\title{
Interference Mitigation for Energy Detection in a Multiband Impulse Radio UWB System
}

\author{
Maximilian Hauske, Holger Jaekel, Hanns-Ulrich Dehner, and Friedrich K. Jondral
}

\begin{abstract}
On-Off-Keying with energy detection is a promising candidate when aiming at simple receiver concepts, due to the fact that simple energy detection reduces receiver complexity. On the other hand, data rates are low and interference mitigation by correlation is no longer possible. This paper proposes and analyzes a method for mitigating asynchronous interferers in energy detection receivers by adapting the maximum likelihood decision rule. It is shown that detection in the presence of interferers is improved and detection in absence of interferers is not declined.
\end{abstract}

Keywords - interference mitigation, energy detection, on-offkeying, multiband impulse radio UWB

\section{INTRODUCTION}

An important advantage of On-Off-Keying (OOK) in combination with energy detection is the possible reduction of receiver complexity. Thus, OOK with energy detection is an interesting candidate for low-complexity systems. Main advantages are robustness against pulse distortions and reduced sensitivity to timing errors. By designing a system with multiple parallel OOK channels higher data rates may be achieved.

Higher data rates can be realized by multiband impulse radio ultra-wideband (UWB) systems which use the frequency range from $3.1 \mathrm{GHz}$ to $10.6 \mathrm{GHz}$, according to $\mathrm{FCC}$ regulations [1]. A multiband impulse radio UWB system is proposed by Paquelet et. al. in [2], [3], and analyzed in [4] with respect to its suitability for higher data rates. The proposed system divides the overall UWB spectrum in subbands and employs OOK within each subband. Data rates of several hundred Mbit/s can be achieved [4] and a multiband impulse radio UWB system possessing low to medium complexity may be a candidate for high data rate applications. This motivates the analysis of such a multiband impulse radio UWB system, requiring analysis of OOK systems.

Obviously, the performance of an OOK based system relies on the ability to decide on the presence or the absence of an intended energy transmission within a given interval. Data decision is performed by measuring the energy within a bit interval and deciding for the transmitted bit by comparing the received energy to a threshold. An important question that has to be addressed is the influence of interference on the detection. The following paper proposes a mitigation scheme for interference mitigation in energy detection which - to the authors' best knowledge - has not yet been considered.

M. Hauske, H. Jaekel, H.-U. Dehner, and F. K. Jondral are with the Institut für Nachrichtentechnik, Universität Karlsruhe $(\mathrm{TH})$, Germany (email: $\{$ hauske, jaekel, dehner, fj\}@ int.uni-karlsruhe.de)
Its performance is analyzed, both theoretically and by simulations. Hereby, most of the following theoretical analyses deal with energy detection and do not address specifics of the above mentioned multiband impulse radio UWB system. These characteristics become important when looking at simulations evaluating the proposed mitigation scheme.

The remainder of the paper is organized as follows: After introducing the system model and describing the effects of interference for energy detection in section II, mitigation of interference is addressed in section III and results of simulations are presented. Section IV concludes the paper.

\section{PRELIMINARIES}

\section{A. System Model}

In OOK a binary zero is represented by transmitting nothing at all, whereas a binary one is represented by transmitting a predefined pulse $s(t)$. Denoting the bit length by $T_{\mathrm{b}}$, when transmitting bits $a_{0}, \ldots, a_{N-1}$ the transmitter signal becomes

$$
x(t)=\sum_{n=0}^{N-1} a_{n} s\left(t-n T_{\mathrm{b}}\right) .
$$

Transmission via a multipath channel results in a receiver pulse

$$
r(t)=\int h(t, \tau) x(t-\tau) \mathrm{d} \tau+n(t),
$$

where $h(t, \tau)$ is the time-variant impulse response of the channel, being a realization of a random impulse response $H(t, \tau) . n(t)$ is a realization of a zero mean, additive white gaussian noise (AWGN) process $N(t)$ with variance $\sigma^{2}$ being related to the single-sided power spectral density $N_{0}$ of the noise by $\sigma^{2}={ }^{N_{0}}$

bandwidth.

$$
{ }_{2} 2 W=N_{0} \cdot W \text { with } 2 W \text { being the system }
$$

Due to the statistical nature of $h(t, \tau)$ and $n(t)$, signal $r(t)$ represents the realization of a random process $R(t)$. For sake of simplicity, no capitals are used in the following paper for random processes, but only the corresponding realizations are dealt with. Anyhow, the statistical nature of all functions has to be kept in mind.

Assuming the channel to be constant during the transmission of a packet, impulse response $h(t, \tau)$ may be considered to be deterministic. This assumption is, for example, justified when analyzing a high data rate short distance link in an office environment, which is an exemplary application of the above mentioned high data rate short range UWB devices.

In order to demodulate the $n^{\text {th }}$ bit the receiver determines

$$
y_{n}=\int_{n T_{\mathrm{b}}}^{n T_{\mathrm{b}}+T} r^{2}(t) \mathrm{d} t, \quad n=0, \ldots, N-1
$$


with $T \leq T_{\mathrm{b}}$ being the integration time which can be chosen with respect to an optimized signal-to-noise ratio (SNR).

If signal and noise are bandlimited to $(-W, W)$, output $r(t)$ is also bandlimited to $(-W, W)$ and may be represented by samples $r(i / 2 W)$, The sampling frequency is independent of the channel impulse response $h(t, \tau)$ : If channel $h(t, \tau)$ is limiting the spectrum to maximum frequency $W^{\prime}<W$ then sampling frequency $2 W$ is still feasible. Thus, in "worst case", calculations are getting too complicated but never incorrect.

Sampling $r(t)$ with rate $1 / 2 W$ within an interval of length $T$ leads to

$$
y_{n} \approx \sum_{\ell=0}^{2 W T-1} \frac{1}{2 W} \cdot r^{2}\left(n T_{\mathrm{b}}+\frac{\ell}{2 W}\right) .
$$

Thus, energy $y_{n}$ possesses a central $\left(a_{n}=0\right)$ or non-central $\left(a_{n}=1\right) \chi^{2}$-distribution with $M=2 W T$ degrees of freedom [5], [6]. ${ }^{1}$ The probability density functions (PDFs) of energy $y_{n}$ in $\left[n T_{\mathrm{b}}, n T_{\mathrm{b}}+T\right)$ for arbitrary but fixed channel impulse response $h(t, \tau)$ are given by

$$
p\left(y_{n} \mid 0, h\right)=\frac{1}{2^{\frac{M}{2}} \Gamma\left(\frac{M}{2}\right)} y_{n}^{\frac{M}{2}-1} e^{-\frac{y}{2}}
$$

and

$$
p\left(y_{n} \mid 1, h\right)=\frac{1}{2}\left(\frac{y_{n}}{E_{n}}\right)^{\frac{M-2}{4}} I_{\frac{M-2}{2}}\left(\sqrt{E_{n} y_{n}}\right) e^{-\frac{y_{n}+E_{n}}{2}}
$$

for $y_{n} \geq 0$, where $E_{n}$ denotes the non-centrality parameter [5] and equals the energy of the received signal, and $I_{(M-2) / 2}(\cdot)$ is the modified Bessel function of the first kind and order $\frac{M-2}{2}$. The mean of the central and non-central $\chi^{2}$-distribution is given by $2 M \sigma^{2}$ and $2 M \sigma^{2}+E_{n}$, respectively.

Maximum likelihood (ML) detection aims at best explaining the observed value by maximizing the conditional PDF of the observation. The ML decision becomes

$$
p\left(y_{n} \mid 0, h\right) \underset{\hat{a}_{n}=1}{\stackrel{\hat{a}_{n}=0}{\gtrless}} p\left(y_{n} \mid 1, h\right) .
$$

Substituting for the PDFs according to equations (5) and (6), and equating $p\left(\gamma_{\mathrm{th}} \mid 0, h\right)=p\left(\gamma_{\mathrm{th}} \mid 1, h\right)$ leads to a threshold $\gamma_{\mathrm{th}}$. ML detection is performed by comparing the received energy $y_{n}$ to threshold $\gamma_{\text {th }}$ and deciding for a transmitted zero if $y_{n}<$ $\gamma_{\mathrm{th}}$ or for a transmitted one if $y_{n} \geq \gamma_{\mathrm{th}}$.

The threshold $\gamma_{\text {th }}$ for optimal ML detection cannot be calculated analytically. In order to solve for threshold $\gamma_{\text {th }}$ the Bessel function is approximated using

$$
I_{\ell}(x) \approx \frac{e^{x}}{\sqrt{2 \pi x}}
$$

which is valid for large arguments $x$ [7], [8]. Paquelet et. al. [2] give also an approximation based on asymptotic approximation of the modified Bessel function, but stop approximation by using a "tabulated function" with restricted validity. Further approximations given in literature are based on gaussian approximations [9].

\footnotetext{
${ }^{1}$ The number of degrees of freedom used in this paper differs from the number given in [2], [3].
}

Definitely, the threshold $\gamma_{\text {th }}$ will depend on the average received energy $E$ and the noise power $N$. Thus, both parameters have to be estimated. In [3], when dealing with incoherent multiband impulse radio UWB, it is suggested to use a preamble of length $m_{0}+m_{1}$ bits, consisting of $m_{0}$ zeros $a_{1}=\ldots=a_{m_{0}}=0$ and $m_{1}$ ones $a_{m_{0}+1}=\ldots=$ $a_{m_{0}+m_{1}}=1$, and to estimate noise power spectral density and signal energy according to:

$$
\widehat{N_{0}}=\frac{1}{m_{0}} \sum_{i=1}^{m_{0}} y_{i}
$$

and

$$
\widehat{E}=\frac{1}{m_{1}} \sum_{i=1}^{m_{1}} y_{m_{0}+i}-\widehat{N_{0}}
$$

Estimation of expected energy levels has significant influence on the data decisions in AWGN. As it will turn out, this estimation is of paramount importance to the system behavior in case of interference, because interferers will lead to an increase in observed signal energy. As a consequence, incorrect estimations of the energy level - and thus the threshold $\gamma_{\text {th }}-$ will adapt, resulting in error propagation and a system breakdown.

For sake of simplicity, the index $n$ referring to the $n^{\text {th }}$ bit interval is suppressed in the following and bit intervals are considered independently. This is justified if bit duration is relatively large compared to the channel and thus inter symbol interference due to the channel can be neglected.

\section{B. Interference in Energy Detection}

Superposition of an asynchronous interference $i(t)$ caused by another device of the same type (i.e., in-band interference) leads to a distorted received signal

$$
r_{\mathrm{d}}(t)=r(t)+i(t)
$$

and thus to

$$
\begin{aligned}
y_{\mathrm{d}} & =\int_{0}^{T} r^{2}(t)+i^{2}(t)+2 r(t) i(t) \mathrm{d} t \\
& =\|r(t)\|^{2}+\|i(t)\|^{2}+2\langle r(t) \mid i(t)\rangle,
\end{aligned}
$$

with $\|x(t)\|^{2}$ being the energy of signal $x(t)$ and $\langle x(t) \mid y(t)\rangle$ denoting correlation of $x(t)$ and $y(t)$. Due to the CauchySchwarz inequality

$$
(\|r(t)\|-\|i(t)\|)^{2} \leq y_{\mathrm{d}} \leq(\|r(t)\|+\|i(t)\|)^{2} .
$$

Depending on inner product $\langle r(t) \mid i(t)\rangle$ three cases can occur: Orthogonal signals $r(t)$ and $i(t)$ lead to an increase of energy which is the energy of interference $i(t)$ within the symbol interval. ${ }^{2}$ If signal and interference interfere constructively, then the increase of energy is larger than in the case of orthogonal interference and the latter becomes a lower bound. Also, the inner product may be negative, corresponding to destructive interference which may reduce overall energy.

If pulses $r(t)$ and $i(t)$ are orthogonal, e.g., if the delay of the interference signal is greater than the pulse duration of $r(t)$

\footnotetext{
${ }^{2}$ Note that interference adds up before squaring. Therefore, even orthogonal interference leads to an increase of energy.
} 
and $i(t)$, the increase of energy corresponds to the interference energy within the integration interval. Orthogonal signals $r(t)$ and $i(t)$ become more and more likely if both signals are generated by devices of the same type both possessing low duty cycle. In this case, probability of non orthogonal signals corresponds to the probability of overlapping signals. With symbol length $T$, pulse length $T_{\mathrm{p}}$ and duty cycle $d=T_{\mathrm{p}} / T$ the probability of non orthogonal signals is at most $2 T_{\mathrm{p}} / T=2 d$, because even the signals are overlapping they may interfere destructively. This is true for multiband impulse radio UWB signals due to their oscillating pulse shape. Thus, the situation of orthogonal signals is addressed in the simulations.

Consequently, assuming orthogonal interference, a binary zero may be mistaken as a binary one whereas a binary one is rarely falsified. However, even if a binary one is very rarely falsified by interference, the estimation of the expected signal energy $E$ may be severely influenced by such an interference. If a very strong interference is superimposed, a very large energy $y \gg E$ is observed. Therefore, the adaptively estimated mean signal energy $E$ and consequently threshold $\gamma_{\text {th }}$ is increased. This may result in multiple bit errors when subsequent binary ones are mistaken for binary zeros.

\section{INTERFERENCE MITIGATION IN ENERGY DETECTION}

\section{A. Proposed Interference Mitigation}

To improve ML detection and to mitigate asynchronous interference it is suggested to subdivide the integration interval $[0, T]$ in $K$ sub-intervals $[0, T / K],[T / K, 2 T / K], \ldots$, each of length $T / K$. This results in $K$ energies

$$
\xi_{k}=\int_{k \frac{T}{K}}^{(k+1) \frac{T}{K}} r_{\mathrm{d}}^{2}(t) \mathrm{d} t, \quad k=0, \ldots, K-1,
$$

with central $\chi^{2}$-distribution $(a=0)$ or a non-central $\chi^{2}$ distribution $(a=1)$ with non-centrality parameter corresponding to the energy in the $k^{t h}$ sub-interval. Note that in noiseonly intervals $(a=0)$ without interferers PDF is independent of index $k$; if interferers are present then PDF depends on the interference energy in the sub-interval.

In this way, $K$ detections are performed simultaneously by comparing $K$ energy values to $K$ thresholds. Consequently, $K$ estimators have to be calculated in parallel which is not too hard using simple moving averages.

Using the proposed mitigation scheme, a "finger-print" is incorporated in the decision and any interference possessing different energy characterization can be mitigated. Thus, the pulse shape and influences of the channel are considered. If the observed energy distribution does not comply with the characteristic energy distribution, either channel characteristics have changed or signals of other sources are severely influencing the observation. Hereby, all slow dynamics of the channel can be controlled by adapting the thresholds in each subinterval.

\section{B. Statistical Properties of Sub-Interval Energies}

An important aspect when dealing with values $\xi_{k}$ is concerning their statistical properties. As already mentioned for energies $y_{n}$, values $\xi_{k}$ are $\chi^{2}$ distributed. Denoting sampling times by $t_{k, \ell}=k \frac{T}{K}+\frac{\ell}{2 W}$ sub-interval energies become

$$
\xi_{k} \approx \sum_{\ell=0}^{\frac{2 W T}{K}-1}\left[\frac{1}{\sqrt{2 W}} r_{\operatorname{det}}\left(t_{k, \ell}\right)+\frac{1}{\sqrt{2 W}} n\left(t_{k, \ell}\right)\right]^{2},
$$

with $r_{\text {det }}(t)$ being the non-random part of $r_{\mathrm{d}}(t)$. Arguments of parenthesis are gaussian distributed with mean $1 / \sqrt{2 W}$. $r_{\text {det }}\left(t_{k, \ell}\right)$ and variance $\sigma^{2} / 2 W$. Thus, sub-interval energy $\xi_{k}$ is generated by squaring and summing up independent gaussian random variables.

When calculating $\xi_{0}, \ldots, \xi_{K-1}$, the $2 W T$ independent samples of the received signal are subdivided into disjoint sets of $2 W T / K$ samples. Due to the fact that functions of pairwise disjoint sets of independent random variables are independent, values $\xi_{0}, \ldots, \xi_{K-1}$ are statistically independent. ${ }^{3}$

If interference signal $i(t)$ has a longer pulse duration than signal $r(t)$, then all sub-interval energies are influenced the same way and the shape of the finger-print remains the same even if its level changes. If, on the other hand, the pulse width of the interference is much smaller than the signal pulse width then only several sub-intervals are influenced and the fingerprint is distorted.

\section{Proposed Modified Decision Rule}

A natural decision rule would be to decide on a binary zero (one) if the majority of sub-intervals decides in favor of a binary zero (one). Nevertheless, this method suffers from the disadvantage that the reliability of sub-decisions is not included in the bit decision, but only hard sub-decisions are used. This method may be improved using soft values which means that the likelihood ratios

$$
\tilde{\lambda}_{k}=\frac{p_{k}\left(\xi_{k} \mid 1, h\right)}{p\left(\xi_{k} \mid 0, h\right)}, \quad k=0, \ldots, K-1,
$$

of all sub-intervals are part of the decision process. Merging of the $K$ sub-decisions, which are assumed to be independent, is realized by multiplication of the likelihood ratios and results in

$$
\prod_{k=0}^{K-1} \tilde{\lambda}_{k} \underset{1}{\stackrel{0}{\lessgtr}} 1
$$

Thus, likelihood ratios representing weak decisions, i.e., $\left|\tilde{\lambda}_{k}\right| \approx 1$, will not influence the overall bit decision too hard, whereas strong decisions, i.e., $\left|\tilde{\lambda}_{k}\right| \ll 1$ or $\left|\tilde{\lambda}_{k}\right| \gg 1$, have considerable influence.

A drawback of this method is the fact that even if both $p(\xi \mid 0, h)$ and $p_{k}(\xi \mid 1, h)$ tend to zero for $\xi \rightarrow \infty$, the ratio $p_{k}(\xi \mid 1, h) / p(\xi \mid 0, h)$ tends to infinity for $\xi \rightarrow \infty$. Thus, strong interferers within one sub-interval may falsify the bit decision by severely increasing the left hand side of equation (18).

Observing that the absolute values of $p(\xi \mid 0, h)$ and $p_{k}(\xi \mid 1, h)$ are both about zero for large $\xi$ and only their ratio is increasing, the problem is solved by adapting the PDFs and

\footnotetext{
${ }^{3}$ This may contradict intuition because the deterministic part of the received signal $r_{\text {det }}(t)$ possesses strong dependencies due to the pulse shape, and thus energies in adjacent intervals are related. Nevertheless, what is considered in further considerations are PDFs which are influenced only by the noise.
} 
adding a positive constant $c>0$ which is larger than zero but which is small with respect to the maximum values of all PDFs:

$$
c \ll \max _{\xi}\left\{p(\xi \mid 0, h), p_{0}(\xi \mid 1, h), \ldots, p_{K-1}(\xi \mid 1, h)\right\} .
$$

The likelihood ratios are modified ${ }^{4}$ to

$$
\lambda_{k}=\frac{p_{k}\left(\xi_{k} \mid 1, h\right)+c}{p\left(\xi_{k} \mid 0, h\right)+c}, \quad k=0, \ldots, K-1,
$$

and decision is made according to

$$
\prod_{k=0}^{K-1} \lambda_{k} \stackrel{0}{\lessgtr} 1
$$

Thus, all values of the likelihood ratio in the expected energy region are not significantly altered whereas very large energy values no longer influence the decision due to $\lambda_{k} \rightarrow 1$ as $\xi \rightarrow \infty$. Strong interferers, which increase the energy level of a sub-interval to an implausible level, are mitigated resp. suppressed.

\section{Discussion of the Proposed Interference Mitigation}

The use of modified likelihood ratios corresponds to a descriptive selection of a region around the expected energy profile of a user. All sub-interval energies differing significantly from the expected value are attenuated by the use of the modified likelihood ratio $\lambda_{k}$. Thus, the proposed method may be illustrated as choosing a confidence region around the finger-print and weighting energies according to their plausibility.

Another thing to notice is the requirement of a stationary user, or at least a user whose channel characteristics change relatively slow in comparison to the bit duration. This is mandatory in order to track changes in its channel characteristics and to not loose the lock on its transmission.

\section{Simulations}

As already mentioned in the introduction, the analyses discussed in this paper originated when analyzing the potential of multiband impulse radio UWB system as a candidate for low-complexity high-data rate devices. Thus, in order to investigate the proposed method for interference mitigation, simulations are carried out with a simulator for an incoherent multiband impulse radio UWB system ([2], [3], [4]).

\section{A. Simulation Results: Interference Mitigation}

In order to separate system effects from effects of energy detection, only one subband of $300 \mathrm{MHz}$ width was considered. Besides the attempt to mitigate effects of the multiband impulse radio UWB system, all simulation results will be influenced by certain characteristics of this system. In order to assess the performance of the proposed mitigation scheme for application in general OOK based systems, further simulations have to be carried out.

\footnotetext{
${ }^{4}$ It should be noticed that modified functions are no longer PDFs.
}

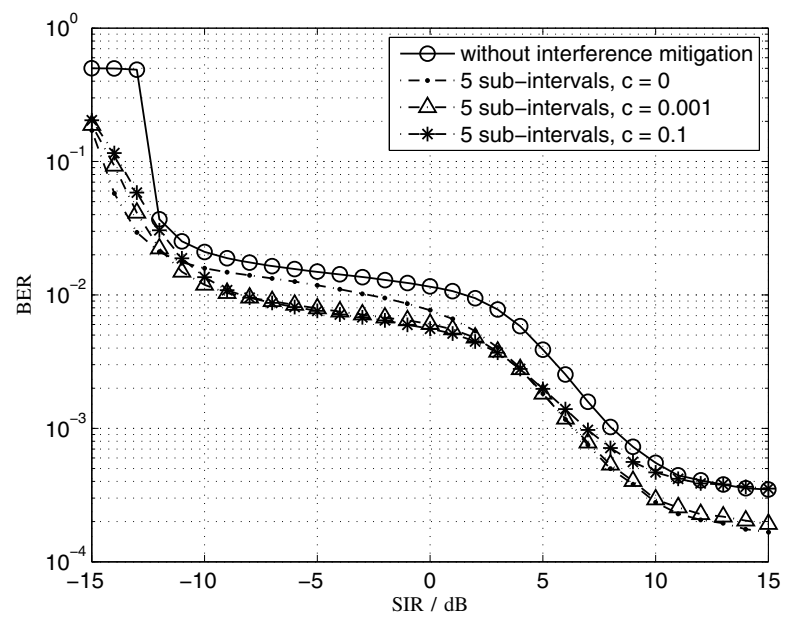

Fig. 1. Simulation results showing that the proposed method improves performance in case of bad SIR and not deteriorates performance in the high SIR region

The system employs OOK within each subband with a data rate of $25 \mathrm{MBit} / \mathrm{s}$ corresponding to $T_{\mathrm{b}}=40 \mathrm{~ns}$. The IEEE channel model 1 (CM1) according to [10] is used. As specified in [10] 100 channel realizations are computed and the best 90 of 100 are used for simulation. Transmission is affected by AWGN; interference is generated by an asynchronous identical multiband impulse radio UWB system of lower data rate $0.75 \mathrm{MBit} / \mathrm{s}$ corresponding to $T_{\mathrm{b}}=1.33 \mathrm{~ms}$. The channel realization of the main system is kept constant during simulation. This leads to an ideal channel estimation without interference and allows to focus on degradation of the estimation through interference. The channel of the interferer cycles through the computed 90 realizations of CM1. The signal-tonoise-ratio is $E_{b} / N_{0}=13 \mathrm{~dB}$, resulting in a bit error rate (BER) of $10^{-4}$ in AWGN. The conditional PDFs of the ML detection given by (5) and (6) are computed numerically. The estimation of the received energy $E$ and the noise power $N$ is realized in two steps. At first an initial estimation is calculated by a preamble using (9) and (10). This estimation is then enhanced recursively during data transmission by using simple moving averages. Three receiver concepts are simulated: a receiver without interference mitigation, a receiver with five sub-intervals and $c=0$, and a receiver using five sub-intervals, $c=0.1$ and $c=0.001$, respectively.

In Fig. 1, the BER is given as a function of the signalto-interference-ratio (SIR). Three characteristic SIR regions are recognized: weak interference (SIR $>10 \mathrm{~dB}$ ), medium interference $(-10 \mathrm{~dB}<\mathrm{SIR}<10 \mathrm{~dB})$ and strong interference (SIR $<-10 \mathrm{~dB})$. For large SIRs all BERs converge to the limit of the undistorted system. With medium interference system performance deteriorates. The s-shaped BERs may be illustrated as the superposition of an interfered and a undistorted BER (two "standard BER shapes": without interferer the system works well, in case of an interfered pulse the bit cannot be received properly.) For a strong interferer (low SIR) system performance drops completely, although only few pulses are actually interfered. The cause of this 


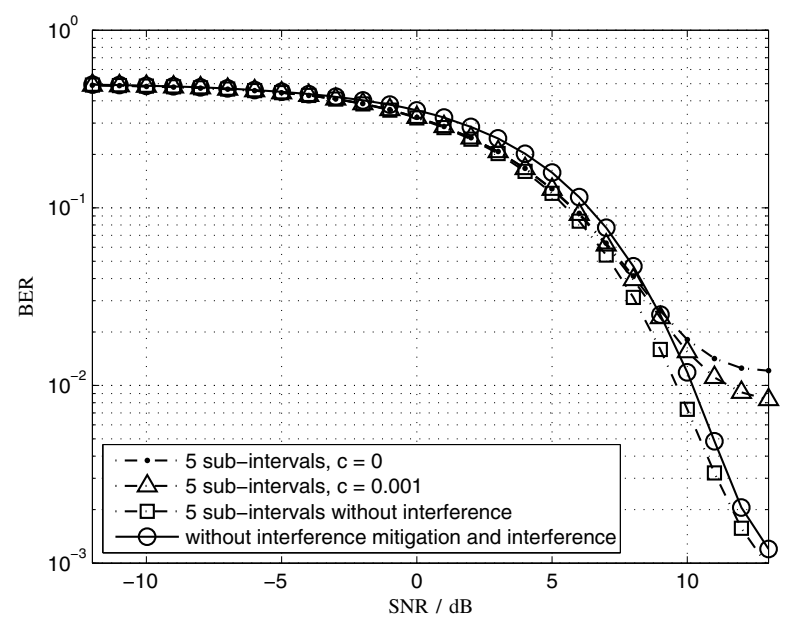

Fig. 2. Simulation results showing the overall system performance of the proposed multiband impulse radio UWB system with and without interference

deterioration is the recursive estimation of the received energy and noise level for detection, which can become unstable. Due to an increased time dependence in case of five subintervals, different methods were used for the estimation of $E$ and $N$, resulting in different deterioration shapes in the low SIR region.

Using five sub-intervals, system performance is enhanced over the whole SIR area. The finger-print-like energy distribution of the signals is used to enhance detection. The use of an additive constant $c$ mitigates strong interferers without worsening the decision in the high SIR region. In case of weak interference the receiver with $c=0.001$ almost reaches the performance of the receiver with the unmodified PDFs $(c=0)$ which should be optimal without interference. In the regions of medium and strong interference, both modified PDFs with $c=0.001$ and $c=0.1$ show a better performance than unmodified detection. The receiver with $c=0.001$ shows the best performance over the whole SIR area with a maximum gain of about $5 \mathrm{~dB}$ compared to the receiver with unmodified PDFs.

\section{B. Simulation Results: Overall System Performance}

To demonstrate the capability of the proposed interference mitigation scheme for a multiband system, simulations with a multiband impulse radio UWB system with 24 subbands were carried out. System parameters and simulation setup are identical to the singleband system above. The whole system uses a bandwidth of $7.5 \mathrm{GHz}$ with an overall data rate of $600 \mathrm{MBit} / \mathrm{s}$.

In Fig. 2 the BER is given as a function of the SNR. The signal-to-interference-ratio (SIR) is set to $-5 \mathrm{~dB}$. Two characteristic regions can be distinguished. For $\mathrm{SNR}<5 \mathrm{~dB}$ AWGN is the dominant effect, resulting in equal BERs with and without interference. For SNR $>5 \mathrm{~dB}$ AWGN gets small compared to interference, resulting in an error floor at $\mathrm{BER} \approx 10^{-2}$, whereas the receiver with the proposed interference mitigation and the modified PDFs shows a slightly better performance. Without interference the system performance is somewhat worse compared to 5 sub-intervals. With five subintervals, the receiver has an additional degree of freedom in weighting and combining the received energy and thus shows a better performance compared to only a single interval.

\section{CONCLUSION}

The paper proposes a method for interference mitigation in incoherent detection of OOK signals in multiband impulse radio UWB systems. Besides the application to multiband impulse radio UWB systems the interference mitigation may also be used for general OOK based transmission systems. In general, there is a trade-off between receiver complexity and interference mitigation. With incoherent detection, receiver complexity can be decreased, but due to the lack of a correlator, the shape of the signal waveform cannot be used for detection. Furthermore, interferers have a crucial influence on the performance of incoherent systems.

In order to mitigate the effect of interference, a modified likelihood ratio was proposed and analyzed. Simulations show that the modified ML detection improves system performance. Nevertheless, only a small enhancement can be achieved and more complex and sensitive estimators are necessary. Further simulations should be carried out in order to separate the influence of the modified ML method from recursive estimation and characteristics of the multiband impulse radio UWB environment.

\section{ACKNOWLEDGMENT}

The authors gratefully acknowledge that their work on interference mitigation in energy detection was partially supported within the priority program No. 1202 (UKoLoS) by the German Research Foundation (DFG).

\section{REFERENCES}

[1] Federal Communications Commission, "Technical Requirements for Indoor UWB Systems," Code of Federal Regulations, no. $47 \S 15.517$, October 2002.

[2] S. Paquelet, L.-M. Aubert, and B. Uguen, "An Impulse Radio Asynchronous Transceiver for High Data Rates," International Workshop on Wideband Systems joint with Conference on Ultrawideband Systems and Technologies. Joint UWBST \& IWUWBS., pp. 18 - 21, May 2004.

[3] S. Paquelet and L.-M. Aubert, "An Energy Adaptive Demodulation for High Data Rates with Impulse Radio," IEEE Radio and Wireless Conference, pp. 323 - 326, September 2004.

[4] M. Mittelbach, R. Moorfeld, and A. Finger, "Performance of a Multiband Impulse Radio UWB Architecture," 3rd IEE Mobility Conference 2006, Bangkok, Thailand, October 2006.

[5] H. Urkowitz, Signal Theory and Random Processes. Dedham: Artech House Inc., 1983.

[6] R. Mills and G. Prescott, "A Comparison of Various Radiometer Detection Models," IEEE Transactions on Aerospace and Electronic Systems, January 1996.

[7] L.-M. Aubert, Mise en place d'une couche physique pour les futurs systemes de radiocommunications hauts debits UWB. These docteur, 2005.

[8] I. Bronstein and K. Semendjaev, Taschenbuch der Mathematik. Harri Deutsch, Thun, 24. Aufl. 1989.

[9] P. A. Humblet and M. Azizoĝlu, "On the Bit Error Rate of Lightwave Systems with Optical Amplifiers," IEEE Journal of Lightwave Technology, vol. 9, no. 11, pp. 1576-1582, 1991.

[10] IEEE P802.15 Working Group for Wireless Personal Area Networks (WPANs), "Channel modeling sub-committee report final, Tech. Rep. IEEE P802.15-02/368r5-SG3a, December 2002. 